Int. J. Contemp. Math. Sciences, Vol. 9, 2014, no. 1, 19 - 23

HIKARI Ltd, www.m-hikari.com

http://dx.doi.org/10.12988/ijcms.2014.26134

\title{
Preserving Sparity for General Solution of Linear Diophantine Systems
}

\author{
Mostafa Khorramizadeh \\ Department of Mathematical Sciences \\ Shiraz University of Technology \\ Shiraz 71555-313, Iran \\ m.khorrami@sutech.ac.ir
}

Copyright (C) 2013 Mostafa Khorramizadeh. This is an open access article distributed under the Creative Commons Attribution License, which permits unrestricted use, distribution, and reproduction in any medium, provided the original work is properly cited.

\begin{abstract}
Here, we present an efficient algorithm for preserveving sparsity in computing the general solution of linear Diophantin systems. In the $k$ th iteration of the algorithm, the general solution $k-1$ equations of the systems is at hand. Then, we present numerical results to justify the efficiency of the resulting algorithm.
\end{abstract}

Mathematics Subject Classification: 11D04, 65Y04

Keywords: linear Diophantine equations, Sparsity, Greatest common divisor, general solution

\section{Introduction}

Consider the linear Diophantine system

$$
A x=b, \quad A \in Z^{m \times n}, x \in Z^{n}, \quad b \in Z^{m}, \quad m \leq n, \operatorname{rank}(A)=m .
$$

By solving (1), we mean finding an integer vector $v \in Z^{n}$ satisfying (1) and an integer matrix $N \in Z^{n \times(n-m)}$ with linearly independent columns so that every $x \in Z^{n}$ satisfies (1) if and only if there exists some $y \in Z^{n-m}$ so that $x=v+N y . v$ is the particular solution and $N$ is a basis for the null space of $A$. Linear Diophantine systems has many applicaions in engineering and 
mathematical sciences such as design of circuits, graph theory, integer rogramming anf etc. There are many algorithms for computing the solution of a linear Diophantine system $[1,2,3,4]$. Many of these algorithms, including those of $[2,4]$, are generalization of algorithms for solving a single linear Diophantine equation such as the extended greatet common divisor [5] and the Rosser's algorithm [7] and etc. This paper is concerned with preserving sparsity in computations of linear Diophantine systems. In section 2 we propose an efficient algorithm for computing the general solution of a linear diophantine equation which preserves sparsity. In section 3, we generaliza the proposed algorithm to obtain an algorthm for computing the general solution of a system of linear Diophantine equations which preserves sparsity. In section 4 we justify the efficiency of our proposed algotithm by presenting some numerical results. Finally, section 5 is devoted to concluding remarks.

\section{Single linear Diophantine equation}

Cosider the following single linearDiophantine equation

$$
a_{1}^{T} x=a_{11} x_{1}+a_{12} x_{2}+\cdots+a_{1 n} x_{n}=b_{1}, \quad a_{1}, x \in Z^{n}, \quad b_{1} \in Z .
$$

Let $v=\left(v_{1}, \cdots, v_{n}\right)^{T} \in Z^{n}$. Throughout the paper, by $\operatorname{gcd}\left(v^{T}\right)$ we mean the greatest common divisor of the elements of $v$. Using the same notations as in [4], let $I$ denote the $n \times n$ identity matrix and

$$
C=\left[\begin{array}{c}
a_{1}^{T} \\
U
\end{array}\right]=\left[\begin{array}{cccc}
a_{11} & a_{12} & \cdots & a_{1 n} \\
1 & 0 & \cdots & 0 \\
0 & 1 & \cdots & 0 \\
\vdots & \vdots & & \vdots \\
0 & 0 & \cdots & 1
\end{array}\right], \quad B=\left[\begin{array}{c}
-b_{1} \\
0
\end{array}\right]=\left[\begin{array}{c}
-b_{1} \\
0 \\
0 \\
\vdots \\
0
\end{array}\right]
$$

Assume $a_{1} \neq 0$. Let $c_{i j}$ be the element in the $i$-th row and $j$-th column of $C$, $C_{j}$ be the $j$-th column of $C$ and $B_{j}$ be the $j$-th component of $B$. Moreover, let $a$ and $b$ be two integer numbers. We say $a$ divides $b$, and write $a \mid b$ if and only if $b=a q$ for some $q \in Z$. Below, $\lfloor x\rfloor$ is the greatest integer number less than or equal to $x$. Our proposed algorithm for solving a single linear Diophantine equation is as follows. Despite other algorithms, this algorithm selects the next two sparsest columns to perform the Euclidean algorithm.

Algorithm 2.1 Sparse linear Diophantine Solver (SDS)

Step $1:$ For $j=1, \cdots, n$ if the leading component of $C_{j}$ is negative then replace $C_{j}$ by $-C_{j}$. Sort $C_{1}, C_{2}, \cdots, C_{n}$ in nonincreasing order with respect to number of nonzero columns of $U$ so that $c_{11}>c_{12}$. 
Step 2 : While $c_{12} \neq 0$ do $\left(B \leftarrow B-\left\lfloor\frac{B_{1}}{c_{11}}\right\rfloor C_{1}, C_{1} \leftarrow C_{1}-\left\lfloor\frac{c_{11}}{c_{12}}\right\rfloor C_{2}\right.$. Sort the columns $C_{1}, C_{2}, \cdots, C_{n}$ in nonincreasing order with respect to to number of nonzero columns of $U$ so that $c_{11}>c_{12}$.)

Step 3 : Set $B \leftarrow B-\left\lfloor\frac{B_{1}}{c_{11}}\right\rfloor C_{1}$. At this point the matrix $C$ and the vector $B$ have the forms:

$$
C=\left[\begin{array}{cc}
\delta & 0 \\
p & U
\end{array}\right]=\left[\begin{array}{cccc}
\delta & 0 & \cdots & 0 \\
p & u_{1} & \cdots & u_{n-1}
\end{array}\right], \quad B=\left[\begin{array}{l}
\theta \\
v
\end{array}\right]
$$

where $v, p$ and $u_{i} \in Z^{n}, 1 \leq i \leq n-1$, and $\delta=\operatorname{gcd}\left(a_{1}^{T}\right)$. If $\theta \neq 0$ then (2) has no integer solution else the general integer solution of (2) is:

$$
x=v+y_{1} u_{1}+y_{2} u_{2}+\cdots+y_{n-1} u_{n-1}=v+U y, \quad y \in Z^{n-1} .
$$

\section{Sparsity in linear Diophantine systems}

In this section we develope an efficient algorithm for computing the genral solution of a system of linear Diophantine system (1). Note that we assume that $\operatorname{rank}(A)=m$. Let

$$
\begin{aligned}
& N^{(0)}=I, N^{(i)}=U^{(1)} \cdots U^{(i)}=N^{(k-1)} U^{(i)}, \quad 1 \leq k \leq m \\
& s^{(0)}=0, s^{(i)}=s^{(i-1)}+N^{(i-1)} v^{(i)}, \quad 1 \leq i \leq m,
\end{aligned}
$$

where $U^{(1)}$ is a basis for the integer null space of $a_{1}^{T}$ and $v^{(1)}$ is an integer solution of $a_{1}^{T} x=b_{1}$, both obtained by an application of SDS to $a_{1}^{T} x=b_{1}, U^{(i+1)}$ is the basis for the integer null space of $a_{i+1}^{T} N^{(i)}$, and $v^{(i+1)}$ is the particular integer solution of the single Diophantine equation,

$$
a_{i+1}^{T} N^{(i)} y^{(i)}=b_{i+1}-a_{i+1}^{T} s^{(i)}
$$

both obtained by an application of SDS. Suppose that $A$ has full row rank and the Diophantine system (1) has an integer solution. It can be verified that $s^{(i)}$ is a particular solution and $N^{(i)}$ is a basis for the integer null space of the first $i$ equations, see [6] for the proof. Here, to consider the sparsity issues, in every iteration, among all rows not considered so far, we choose the row with smallest numer of nonzeros for computing $s_{i}$. The resulting algorithm is as follows.

Algorithm 3.1 Sparse linear Diophantine System Solver (SDSS)

Step 1: Set $i=1, v=0, U=I_{n}$. 
Step 2: Among all rows of $A$ not considered so far, let $a_{t_{i}}$ be the row with minimum number of nonzero components. Compute $s_{i}=U^{T} a_{i}$ and $\tau_{i}=$ $b_{i}-a_{i}^{T} v$.

(a) If $s_{i}=0$ and $\tau_{i} \neq 0$ then stop $\{$ the system has no integer solution $\}$

(b) If $s_{i}=0$ and $\tau_{i}=0$ the ith equation is redundant then go to Step 4 .

(c) If $s_{i} \neq 0$ then let

$$
C=\left(\begin{array}{c}
s_{i}^{T} \\
U
\end{array}\right), \quad B=\left(\begin{array}{c}
-b_{i}+a_{i}^{T} v \\
v
\end{array}\right)
$$

Apply $S D S$ to the matrix $C$ and the vector $B$ above. The resulting matrix $C_{\text {fin }}$ and the resulting integer vector $B_{\text {fin }}$ have the forms,

$$
C_{f i n}=\left(\begin{array}{cc}
\delta & 0 \\
p & N
\end{array}\right), \quad B_{f i n}=\left(\begin{array}{c}
\theta \\
s
\end{array}\right) .
$$

If $\theta \neq 0$ then stop the linear Diophantine system $A x=b$ has no integer solution\} else set $v=s, U=N$ and $r=r+1$.

Step 3: Set $v=v+U v^{(i)}, U=U U^{(i)}$.

Step 4: If $i=m$ then stop ( $v$ is an integer solution for (1) and $U$ is a basis for the integer null space of $A$ and $r$ is the rank of $A$ ) else set $i=i+1$ and go to Step 2.

\section{Numerical results}

In this section we provide with a numerical example, showing that our proposed algorithm is efficient. We implemented the algoritm in the Matlab environment, using an Intel Core 2 Duo cpu, $1.8 \mathrm{GHz}$ processor of 1GB RAM. We applied the resulting algorithm to a sparse test problem which is made as follows. We first considered a sparse integer matrix which is given by Mark Giesbrecht and can be obtained from sparse matrix collection of the university of Florida. The matrix has 18 rows and 30 columns. Moreover, the density of the given spare matrix is 0.12 . We then let $x$ be an arbitrary sparse integer vector and compute $b=A x$. This way we are sure that the linear Diophantine system $A x=b$ has sparse integer solutions. We then applied our implemented algorithm to the resulting linear Diophantine system to obtain a null space basis and a particular solution. We obtained an sparse null space basis with 12 rows and 30 columns and a sparse integer solution. The density of the 
resulting null space basis was 0.14 which is close to the density of the original coefficient matrix. To compare our proposed algorithm with an efficient algorithm of the literature we also applied the LLL-based Hermite normal form algorithm [5] to the same linear Diophantine system and obtaned a null space basis with sparsity of 0.21 . In a similar way the sparsity of the particular solution obtained by using our approach is more than that of the LLL-based Hermite normal form algorithm. This justifies the efficiency of our proposed lagorithm.

\section{Conclusions}

In this paper we proposed an efficient algorithm for computing the general solution of a sparse linear Diophantine system and presented numerical results to justify its efficiency. We also compared our proposed algorithm with an algorithm which is based on LLL-reduction algorthms.

ACKNOWLEDGEMENTS. The authors would like to thank the research council of Shiraz university of technology for its support.

\section{References}

[1] W. A. Blankinship, Algorithm 287, matrix triangulation with integer arithmetic [F1], Comm. ACM 9 (1966) 513.

[2] W. A. Blankinship, Algorithm 288, solution of simultaneous linear Diophantine equations, Comm. ACM 9 (1966) 514.

[3] G. H. Bradley, Algorithms for Hermite and Smith normal matrices and linear Diophantine equations, Math. Comp. 25 (1971) 897-907.

[4] T. J. Chou and E. E. Collins, Algorithms for the solutions of systems of linear Diophantine equations, SIAM J. Comput. 11 (1982) 686-708.

[5] G. Havas, B. S. Majewski and K. R. Matthews, Extended GCD and hermite normal form algorithms via lattice basis reduction, Experimental Mathematics 7 (1988) 125-135.

[6] M. Khorramizadeh and N. Mahdavi-Amiri , Integer extended $A B S$ algorithms and possible control of intermediate results for linear Diophantine systems, to appear in 4OR, DOI 10-1007/s 10288-008-0082-8, 23 pages.

[7] J. B. Rosser, A note on the linear Diophantine equation, Amer. Math. Monthly 48 (1941) 662-666.

Received: June 1, 2013 